\title{
Effect of Different Pre Sowing Treatments on Seed Germination and Growth of Custard Apple (Annona squamosa L.) Seedlings
}

\author{
$\operatorname{Deeksha}^{1 *}$, T. R. Sharma ${ }^{1}$, U. K. Chanderia ${ }^{2}$ and B. S. Dwivedi ${ }^{1}$ \\ ${ }^{1}$ Department of Horticulture, College of Agriculture, JNKVV, Jabalpur (M.P.), India \\ ${ }^{2}$ Department of Soil Science and Agricultural Cultural Chemistry, \\ JNKVV Jabalpur (M.P.), India \\ *Corresponding author
}

\begin{tabular}{|l|}
\hline Ke y w o r d s \\
$\begin{array}{l}\text { Seed germination, } \\
\text { Pre sowing } \\
\text { treatments, } \\
\text { Custard apple }\end{array}$ \\
\hline Article Info \\
\hline $\begin{array}{l}\text { Accepted: } \\
15 \text { October } 2020 \\
\text { Available Online: } \\
10 \text { November } 2020\end{array}$ \\
\hline
\end{tabular}

\section{A B S T R A C T}




\section{Introduction}

Custard apple (Annona squamosa L.) belongs to family Annonaceae. The family annonaceae belongs to suborder Mangolineae, Myristicaceae and lauraceae. It is one of the finest fruit gifted to India by tropical America. It has several synonyms such as SitafalSharifa, Sugar apple, Sweet sop etc, Custard apple is generally used as fresh pulp of fruit is juicy, cream, yellow, or white, sweet with pleasant texture and flavour. It contains 28.6-36.9\% edible portion consisting of $12.4-16.6 \%$ sugar, 73.30 moisture, $1.60 \%$ protein, $0.30 \%$ fat, $0.70 \%$ mineral matter, $23.90 \%$ carbohydrate, $0.20 \%$ calcium, $0.40 \%$ phosphorus, $1.0 \%$ iron and $0.26-0.65 \%$ acidity with caloric value of $105 \mathrm{~K}$ cal $/ 100 \mathrm{~g}$ besides high nutritive value, it has also a high medicinal value un .

The fruits are often scaly and succulent and are sometimes segmented. Annonas are mostly grown by seeds either for seedling plants or for vegetative propagation but it can also be propagate by inarching, budding and grafting. There are not common cultivars of this fruit, but it can be classified according to their yellowish and brownish skin colours. The tree is fast growing in nature so, responded positive to the cultural practices i.e. mulching, organic fertilizers, frequent irrigation etc. The fruiting of plant starts after two to three years, plant hold fruit two to three months for repening process. The seeds of Annonaceae are albuminous ellipsoids and their length varies between 5 and $30 \mathrm{~mm}$. ripe fruits, seeds, leaf and root are considered as medicines.

Seed germination is the first stage of plant development, it is the resumption of active growth of embryo that results in the emergence of the young plant. Dormancy in seeds may be due to presence of hard and impermeable seed coat, germination inhibitors and improper development of embryo. To get higher and proper germination seed needs pre sowing treatments which helps in promotion of early and higher percentage of seed germination with healthy vigorous seedling, Seed germination is affected by many factors, which include type of substrate used, environmental factors such as oxygen, water, temperature, and light. Seed without use of growth regulators showed poor response to germination and growth. In recent year, lots of research work has been done on different aspects of growth regulators. The important aspects among them are the most appropriate growth regulators for encouraging germination and further growth. The prices of the growth regulators have sky high so the nursery man who is the regular consumer can't afford it much longer. To overcome this crisis some alternative for growth regulators should be brought up which may be as efficient as growth regulators. Easy to access and cheap. This has diverted the attention once again towards Can sugar, cow urine, bioinoculants (Rhizobacteria).

Cow urine proving feasible may bring a breakthrough in the present context as it is free of cost and easily available through it is not much consistent. Cow urine contains Nitrogen, Sulphur Ammonia, Copper, Iron, Urea, Uric Acid, Phosphorus, Sodium, Potassium, Manganese, Carbolic Calcium, Salt, Vitamins, Lactose, Enzyme, Water, Creatin, Aurum Hydroxide etc. (Dilrukshi, 2009). The enough work has been done on seed germination with use of PGR's and growing media but there is meager work on seed treatment with organic matter like cow urine with soaking period and incorporation of plant growth promoting Rhizobacteria in growing media. In this context, the utilization of plant growth promoting Rhizobacteria (PGPR) may help to develop an environmentally benign biological approach for managing fungal diseases and enhancing the plant health resulting increase the yield. 


\section{Materials and Methods}

The experiment was conducted at vegetable Research Farm, Maharajpur, Department of Horticulture, JNKVV, Jabalpur (M.P.) during 2018-2019. Jabalpur is situated in "Kymore Platue and Satpura Hills" Agro-climatic zone of Madhya Pradesh at $23.9^{\circ}$ North latitude and $79.58^{\circ}$ east longitudes and an altitude of 411.78 meter above the mean sea level. In the experiment the response of different concentration of cow urine, soaking duration and growing media (PGPR) were studied for seed germination, growth and survival of Custard apple seedlings. The experiment was laid out in Asymmetrical Factorial Randomized Block Design with 54 treatment combinations replicated thrice, Observations were recorded using standard procedure and statistically analysed (Table 1).

Total no. of seeds germinated Germination $(\%)=$

Total no of seeds sown

$\mathrm{CVG}=\mathrm{N} 1+\mathrm{N} 2 \ldots+\mathrm{Ni} / 100 \mathrm{X} \mathrm{N} 1 \mathrm{~T} 1 \ldots . .+\mathrm{Ni} \mathrm{Ti}$

Total Leaf Area

LAI = ------------------

\section{Results and Discussion}

\section{Growth parameter}

The following observations were recorded at different time intervals after sowing. Randomly selected five plants were tagged for following observations.

\section{$\%$ of germination at 30 and 60 DAS}

Data pertaining germination at 30 and 60 days has given in table 2 and Data revealed that cow urine had showed significant effect on percent of germination. The maximum germination percent of 44.8 and 70.4 was recorded under cow urine concentration $(20$ $\%) \mathrm{G}_{1}$ at 30 and 60 days after sowing, respectively. minimum germination percent of 39.3 and 56.7 was recorded under seed soaked in tap water $\left(\mathrm{G}_{0}\right)$ after sowing. It is apparent from analysed data that soaking duration significantly affected the percent of germination. The maximum germination percent 43.1 and 61.3 was recorded under $S_{1}$ (seed soaked for $24 \mathrm{hrs}$ ) while, minimum germination percent of 40.8 and 58.1 was recorded under $\mathrm{S}_{3}$ (seed soaked for 72 hrs).The growing media in rich with PGPR had showed the significant effect on germination. The maximum germination percentage of 42.6 and 62.5 was recorded under $\mathrm{P}_{3}$ (6\%). Whereas, minimum germination of 41.6 and 60.4 was recorded under $\mathrm{P}_{1}$ at 30 and 60 days after sowing. Concentration of cow urine and soaking duration had showed significant effect on germination and maximum germination percent of 47.0 and 75.3 was recorded under seed soaked in $20 \%$ cow urine for 24 hours $\left(\mathrm{G}_{1} \mathrm{~S}_{1}\right)$ while, minimum Germination percentage of 38.5 and 55.5 was recorded under $\mathrm{G}_{0} \mathrm{~S}_{3}$ at 30 and 60 days after seed sowing, respectively.

Combine effect of cow urine and growing media (PGPR) had showed significant effect on germination and the maximum germination percentage of 45.5 and 75.3 was recorded at 30 and 60 days after sowing, respectively under $\mathrm{G}_{1} \mathrm{P}_{3}$. While minimum germination percent of 39.1 and 56.2 was recorded under $\mathrm{G}_{0} \mathrm{P}_{1}$ at 30 and 60 days after sowing, respectively.

\section{Coefficient velocity of germination}

The data presented in table 3 indicated that the cow urine and growing media had showed significant effect on coefficient velocity of germination. Whereas, soaking duration had 
not shown any significant effect on coefficient velocity of germination. The maximum coefficient velocity of 2.7 was observed under seed soaked in cow urine concentration (20\%) and minimum 2.1 seed /day under tap water. As regards soaking duration had showed non- significant effect on coefficient velocity of germination. As regards PGPR in rich with growing media had showed significant effect on coefficient velocity of germination. The maximum coefficient velocity of germination 2.4 was noted under $\mathrm{P}_{3}$ and whereas, minimum value of 2.3 was noted under control $\left(\mathrm{P}_{1}\right)$. As regards interaction of cow urine and soaking duration had showed non - significant effect on coefficient velocity of germination .Interaction of cow urine and growing media had showed significant effect on coefficient velocity of germination. The maximum coefficient velocity of germination 2.7 was noted under $\mathrm{G}_{1} \mathrm{P}_{3}$ and whereas, minimum value of 2.3 was noted under $\mathrm{G}_{0} \mathrm{P}_{1}$. Interaction of cow urine, soaking duration and growing media showed non-significant effect on CVG while the maximum $\mathrm{CVG}$ was recorded under $\mathrm{G}_{1} \mathrm{~S}_{1} \mathrm{P}_{3}$.

\section{Height of shoot at 60, 90, 120 and 150 days}

The height of shoots at 60, 90, 120, 150 days after sowing was recorded and presented in Table. Data showed that the cow urine showed significant effect on height of shoots at all the stages. The maximum shoot height of 5.0, 7.3, 12.1 and $13.3 \mathrm{~cm}$ was noted under cow urine concentration of $(20 \%)$. minimum shoot height was recorded under $\left(\mathrm{G}_{0}\right)$ seed soaked in tap water at 60, 90, 120 and 150 days after seed sowing respectively. Cow urine soaking concentration (20\%) showed superiority over rest of the concentrations. As regards to soaking duration had showed significant effect on height of shoot and maximum height of 5.0, 6.8, 11.1 and $13.4 \mathrm{~cm}$ was noted under $\mathrm{S}_{1}$ i.e. seed soaked for $24 \mathrm{hrs}$ of soaked duration at 60,90, 120 and 150 days after seed sowing, respectively and found significantly superior over rest of the duration of seed soaking duration whereas, the minimum height of shoots 4.0, 6.2, 9.8 and $12.0 \mathrm{~cm}$ was recorded under $\mathrm{S} 3$ at 60,90 , 120 and 150 days after seed sowing, respectively.

Inoculation with PGPR in growing media had showed significant effect on height of shoot at various stages. The maximum height of 4.7 , $6.6,10.8,12.9$ was noted under seed grown in PGPR rich $(6 \%)$ growing media at $60,90,120$ and 150 days after sowing, respectively. whereas, the minimum value noted under control $\left(\mathrm{P}_{1}\right)$. Interaction effect of cow urine $\mathrm{x}$ soaking duration had showed significant effect and maximum shoot height recorded under $\mathrm{G}_{1} \mathrm{~S}_{1} \quad(5.2, \quad 7.8,12.6$ and $13.9 \mathrm{~cm})$ whereas, the minimum shoot height was 3.5, $5.9, .6,10.5 \mathrm{~cm}$ was recorded under seed soaked for 72 hours in tap water at 60,90,120 and 150 days after sowing. As regards the cow urine and growing media had showed significant effect on height of shoot and the maximum shoot was of $5.4,7.5,12.3$ and 13.6 $\mathrm{cm}$ under $\mathrm{G}_{1} \mathrm{P}_{3}$ whereas, minimum shoot height of 3.8, 6.0, 8.9 and $10.9 \mathrm{~cm}$ under $\mathrm{G}_{0} \mathrm{P}_{1}$ respectively at 60,90,120 and 150 days after sowing.

Interaction effect of soaking duration and growing media had significant effect on height of shoot at various stages and maximum shoot height noted under $\mathrm{S}_{1} \mathrm{P}_{3}$ 5.7, $7.0,12.2$ and $13.8 \mathrm{~cm}$ was recorded under seed soaked for $24 \mathrm{hrs}$ and growing media in rich with 6\% PGPR. Whereas, the minimum shoot height was observed under $\mathrm{S}_{3} \mathrm{P}_{1}$. Combine effect of cow urine, soaking duration and growing media had showed significant effect on shoot height at various stages and the maximum shoot height was recorded under treatment combination of cow urine concentration (20\%), soaking duration 
(24 hrs), and growing media in rich with PGPR $(6 \%)$.

Length of seedlings at 150 days after sowing

The significantly maximum height of seedling $(24.4 \mathrm{~cm})$ at 150 DAS was recorded under the treatment $\mathrm{G}_{1}$. The present investigations are in conformity with the results of Shristava and Bhel (2002). Probable reasons may be that the cow urine which induced the cell elongation process and ultimately increased the height of the plant. These results are supported by Rao (1975) and Parmar et al., The maximum plant growth (23.84) in PGPR was possibly due to some plant growth promoting rhizobacteria (pseudomonas) that is increase physiological activities of seed, essential for cell division and cell enlargement. Krishnamoothy and Vajranrabhaian (1986) who reported that some plant growth promoting rhizobacteria releases such as cytokinins and auxins like substances. It also contains rich source of nutrient. The results obtained were closely related with the finding of Kumar and Sharma 2007 in Jatropa curcus. As regard to cow urine+ soaking duration + PGPR interaction combinedly affected a significant increase in length of seedlings $(29.8 \mathrm{~cm})$ at 150 days after sowing was observed. Due to inter-nodal cell elongation, there by leading to increase in seedling length. These findings are supported by Ratan and Reddy (2004); Parmar et al., 2016 (Table 5).

\section{Seedling vigour index I and seedling vigour index II}

The data reveals the significant effect of organic sources on seedling vigour index I and seedling vigour index II. The seedling vigour index I (1090.74 $\mathrm{cm}$ and $69.8 \mathrm{~g})$ was recorded under $\mathrm{G}_{1} \mathrm{~S}_{1} \mathrm{P}_{3}$ which is superior to all other combination of cow urine. It is followed by cow urine + soaked duration/cow urine + PGPR concentration. The findings are supported by Gurung et al., (2014).

\section{Energy interception}

In the present study, all the treatment growing media, gibberellic acid concentration and their combination effect showed significant effect on the experiment concern. The probable reason may be that Interception of light by a crop canopy is strongly related to total leaf area. A crop will thus intercept more PAR and hence grow faster if it develops leaf area rapidly. Similar findings were reported by Maddonni and Otegui (1996) (Table 4 and 6).

Table.1 Details of experiment

\begin{tabular}{|l|c|c|}
\hline Factor A & Factor B (Soaked Time) & Factor C (PGPR) \\
\hline $\mathbf{G}_{\mathbf{0}}$ Tap Water-100ml & $\mathrm{S}_{1} 24$ hours & $\mathrm{P}_{1} 0 \%$ \\
\hline $\mathbf{G}_{\mathbf{1}}$ Cow Urine-20\% & $\mathrm{S}_{2} 48$ hours & $\mathrm{P}_{2} 3 \%$ \\
\hline $\mathbf{G}_{\mathbf{2}}$ Cow Urine-40\% & $\mathrm{S}_{3} 72$ hours & $\mathrm{P}_{3} 6 \%$ \\
\hline $\mathbf{G}_{\mathbf{3}}$ Cow Urine-60\% & & \\
\hline $\mathbf{G}_{\mathbf{4}}$ Cow Urine-80\% & & \\
\hline $\mathbf{G}_{\mathbf{5}}$ Cow Urine-100\% & & \\
\hline
\end{tabular}


Table.2 Effect of seed soaking duration, concentration of cow urine and growing media on germination at 30 and 60 DAS (\%)

\begin{tabular}{|c|c|c|c|c|}
\hline \multirow[t]{2}{*}{ Treatment } & \multicolumn{3}{|c|}{ Growing media } & \multirow[t]{2}{*}{ Mean } \\
\hline & Without PGPR $\left(\mathbf{P}_{1}\right)$ & $\begin{array}{c}\text { With PGPR } \\
\left(\mathbf{P}_{2}\right)\end{array}$ & $\begin{array}{c}\text { With PGPR } \\
\left(\mathbf{P}_{3}\right)\end{array}$ & \\
\hline Soaking duration $-24 \mathrm{hr}\left(\mathrm{S}_{1}\right)$ & 60.7 & 61.3 & 62.0 & 61.3 \\
\hline Soaking duration $-48 \mathrm{hr}\left(\mathrm{S}_{2}\right)$ & 59.3 & 59.6 & 60.4 & 59.8 \\
\hline Soaking duration $-72 \mathrm{hr}\left(\mathrm{S}_{3}\right)$ & 58.7 & 56.8 & 58.9 & 58.1 \\
\hline \multirow[t]{2}{*}{ Mean } & 60.0 & 60.1 & 61.4 & \\
\hline & Growing media $(\mathrm{P})$ & \multicolumn{2}{|c|}{ Soaking duration (S) } & $\mathrm{S} \times \mathrm{P}$ \\
\hline SEm \pm & 0.079 & \multicolumn{2}{|c|}{0.079} & 0.136 \\
\hline CD at $5 \%$ & 0.221 & \multicolumn{2}{|c|}{0.221} & 0.382 \\
\hline \multicolumn{5}{|c|}{ Growing media } \\
\hline & Without PGPR $\left(\mathbf{P}_{1}\right)$ & $\begin{array}{c}\text { With PGPR } \\
\left(\mathbf{P}_{2}\right)\end{array}$ & $\begin{array}{c}\text { With PGPR } \\
\left(\mathbf{P}_{\mathbf{3}}\right)\end{array}$ & Mean \\
\hline Tape water $\left(\mathbf{G}_{0}\right)$ & 56.2 & 56.8 & 57.2 & 56.7 \\
\hline Cow urine Conc.- $20 \%\left(\mathbf{G}_{1}\right)$ & 61.6 & 62.6 & 75.3 & 70.4 \\
\hline Cow urine Conc.- $40 \%\left(G_{2}\right)$ & 61.3 & 62.2 & 62.1 & 62.4 \\
\hline Cow urine Conc.- $60 \%\left(G_{3}\right)$ & 59.6 & 60.0 & 60.2 & 60.8 \\
\hline Cow urine Conc.- $80 \%\left(G_{4}\right)$ & 58.3 & 59.3 & 59.2 & 59.2 \\
\hline Cow urine Conc.- $100 \%\left(G_{5}\right)$ & 56.2 & 57.5 & 58.2 & 57.5 \\
\hline \multirow[t]{2}{*}{ Mean } & 60.0 & 60.1 & 61.4 & \\
\hline & Growing media $(\mathrm{P})$ & \multicolumn{2}{|c|}{ Concentration $(\mathrm{G})$} & $\mathrm{G} \times \mathrm{P}$ \\
\hline SEm \pm & 0.079 & \multicolumn{2}{|c|}{0.111} & 0.193 \\
\hline CD at $5 \%$ & 0.221 & \multicolumn{2}{|c|}{0.312} & 0.558 \\
\hline \multicolumn{5}{|c|}{ Soaking Duration } \\
\hline & $24 \mathrm{hr}\left(\mathrm{S}_{1}\right)$ & $48 \mathrm{hr}\left(\mathrm{S}_{2}\right)$ & $72 \mathrm{hr}\left(\mathrm{S}_{3}\right)$ & Mean \\
\hline Tape water $\left(\mathbf{G}_{0}\right)$ & 58.2 & 56.5 & 55.5 & 56.7 \\
\hline Cow urine Conc.- $20 \%\left(G_{1}\right)$ & 75.3 & 64.1 & 62.4 & 70.4 \\
\hline Cow urine Conc.- $40 \%\left(G_{2}\right)$ & 64.1 & 63.2 & 60.8 & 62.4 \\
\hline Cow urine Conc.- $60 \%\left(G_{3}\right)$ & 61.6 & 59.5 & 58.5 & 60.8 \\
\hline Cow urine Conc.- $80 \%\left(\mathbf{G}_{4}\right)$ & 60.7 & 59.6 & 57.4 & 59.2 \\
\hline Cow urine Conc.- $100 \%\left(G_{5}\right)$ & 59.0 & 57.2 & 56.3 & 57.5 \\
\hline \multirow[t]{2}{*}{ Mean } & 61.3 & 59.8 & 58.1 & \\
\hline & Duration (S) & \multicolumn{2}{|c|}{ Concentration $(\mathrm{G})$} & $\mathrm{G} \times \mathrm{S}$ \\
\hline SEm \pm & 0.079 & \multicolumn{2}{|c|}{0.111} & 0.193 \\
\hline CD at $5 \%$ & 0.221 & \multicolumn{2}{|c|}{0.312} & 0.540 \\
\hline
\end{tabular}




\begin{tabular}{|c|c|c|c|c|}
\hline \multirow[t]{2}{*}{ Treatment } & \multicolumn{3}{|c|}{ Growing media } & \multirow[t]{2}{*}{ Mean } \\
\hline & Without PGPR $\left(\mathbf{P}_{1}\right)$ & $\begin{array}{c}\text { With PGPR } \\
\left(\mathbf{P}_{2}\right)\end{array}$ & $\begin{array}{c}\text { With PGPR } \\
\left(\mathbf{P}_{3}\right)\end{array}$ & \\
\hline Soaking duration $-24 \mathrm{hr}\left(\mathrm{S}_{1}\right)$ & 43.1 & 43.3 & 44.2 & 43.1 \\
\hline Soaking duration $-\mathbf{4 8} \mathbf{~ h r}\left(\mathrm{S}_{2}\right)$ & 41.5 & 41.8 & 42.3 & 41.9 \\
\hline Soaking duration $-72 \mathrm{hr}\left(\mathrm{S}_{3}\right)$ & 40.3 & 40.7 & 41.3 & 40.8 \\
\hline \multirow[t]{2}{*}{ Mean } & 41.6 & 41.9 & 42.6 & \\
\hline & Growing media $(\mathrm{P})$ & \multicolumn{2}{|c|}{ Soaking duration (S) } & $S \times P$ \\
\hline SEm \pm & 0.091 & \multicolumn{2}{|c|}{0.091} & 0.157 \\
\hline CD at $5 \%$ & 0.254 & \multicolumn{2}{|c|}{0.254} & 0.471 \\
\hline \multicolumn{5}{|c|}{ Growing media } \\
\hline & Without PGPR $\left(\mathbf{P}_{1}\right)$ & $\begin{array}{c}\text { With PGPR } \\
\left(\mathbf{P}_{2}\right)\end{array}$ & $\begin{array}{c}\text { With PGPR } \\
\left(\mathbf{P}_{\mathbf{3}}\right)\end{array}$ & Mean \\
\hline Tape water $\left(\mathbf{G}_{\mathbf{0}}\right)$ & 39.1 & 39.3 & 39.6 & 39.3 \\
\hline Cow urine Conc.- $20 \%\left(G_{1}\right)$ & 44.4 & 44.5 & 45.5 & 44.8 \\
\hline Cow urine Conc.- $40 \%\left(G_{2}\right)$ & 43.0 & 43.5 & 44.0 & 43.1 \\
\hline Cow urine Conc.- $60 \%\left(G_{3}\right)$ & 42.2 & 42.5 & 43.5 & 42.7 \\
\hline Cow urine Conc.- $80 \%\left(G_{4}\right)$ & 41.0 & 41.1 & 42.0 & 41.4 \\
\hline Cow urine Conc.- $100 \%\left(G_{5}\right)$ & 39.8 & 40.3 & 41.1 & 40.4 \\
\hline \multirow[t]{2}{*}{ Mean } & 41.6 & 41.9 & 42.6 & \\
\hline & Growing media $(\mathrm{P})$ & \multicolumn{2}{|c|}{ Concentration (G) } & $G \times P$ \\
\hline SEm \pm & 0.091 & \multicolumn{2}{|c|}{0.128} & 0.222 \\
\hline CD at $5 \%$ & 0.254 & \multicolumn{2}{|c|}{0.359} & 0.622 \\
\hline \multicolumn{5}{|c|}{ Soaking Duration } \\
\hline & $24 \mathrm{hr}\left(\mathrm{S}_{1}\right)$ & $48 \mathrm{hr}\left(\mathrm{S}_{2}\right)$ & $72 \mathrm{hr}\left(\mathrm{S}_{3}\right)$ & Mean \\
\hline Tape water $\left(\mathbf{G}_{\mathbf{0}}\right)$ & 40.3 & 39.2 & 38.5 & 39.3 \\
\hline Cow urine Conc.- $20 \%\left(G_{1}\right)$ & 47.0 & 44.3 & 44.2 & 44.8 \\
\hline Cow urine Conc.- $40 \%\left(G_{2}\right)$ & 44.7 & 43.3 & 42.4 & 43.1 \\
\hline Cow urine Conc.- $60 \%\left(G_{3}\right)$ & 44.7 & 42.4 & 41.4 & 42.7 \\
\hline Cow urine Conc.- $80 \%\left(G_{4}\right)$ & 42.5 & 41.4 & 41.0 & 41.4 \\
\hline Cow urine Conc.- $100 \%\left(G_{5}\right)$ & 41.6 & 40.3 & 39.3 & 40.4 \\
\hline \multirow[t]{2}{*}{ Mean } & 43.1 & 41.9 & 40.8 & \\
\hline & Duration $(\mathrm{S})$ & \multicolumn{2}{|c|}{ Concentration (G) } & GxS \\
\hline SEm \pm & 0.091 & \multicolumn{2}{|c|}{0.128} & 0.222 \\
\hline CD at $5 \%$ & 0.254 & \multicolumn{2}{|c|}{0.359} & 0.622 \\
\hline
\end{tabular}


Table.3 Effect of seed soaking duration, concentration of cow urine and growing media on Coefficient velocity of germination

\begin{tabular}{|c|c|c|c|c|}
\hline \multirow[t]{2}{*}{ Treatment } & \multicolumn{3}{|c|}{ Growing media } & \multirow[t]{2}{*}{ Mean } \\
\hline & Without PGPR $\left(\mathbf{P}_{1}\right)$ & $\begin{array}{c}\text { With PGPR } \\
\left(\mathbf{P}_{2}\right)\end{array}$ & $\begin{array}{c}\text { With PGPR } \\
\qquad\left(\mathbf{P}_{\mathbf{3}}\right)\end{array}$ & \\
\hline Soaking duration $-24 \mathrm{hr}\left(\mathrm{S}_{1}\right)$ & 2.2 & 2.3 & 2.4 & 2.3 \\
\hline Soaking duration $-48 \mathrm{hr}\left(\mathrm{S}_{2}\right)$ & 2.3 & 2.3 & 2.3 & 2.3 \\
\hline Soaking duration $-72 \mathrm{hr}\left(\mathrm{S}_{3}\right)$ & 2.2 & 2.3 & 2.3 & 2.2 \\
\hline \multirow[t]{2}{*}{ Mean } & 2.3 & 2.3 & 2.4 & \\
\hline & Growing media $(\mathrm{P})$ & \multicolumn{2}{|c|}{ Soaking duration (S) } & $\mathrm{S} \times \mathrm{P}$ \\
\hline SEm \pm & 0.012 & \multicolumn{2}{|c|}{0.012} & 0.021 \\
\hline CD at $5 \%$ & 0.034 & \multicolumn{2}{|c|}{ NS } & NS \\
\hline \multicolumn{5}{|c|}{ Growing media } \\
\hline & Without PGPR $\left(\mathbf{P}_{1}\right)$ & $\begin{array}{c}\text { With PGPR } \\
\left(\mathbf{P}_{2}\right)\end{array}$ & $\begin{array}{c}\text { With PGPR } \\
\left(\mathbf{P}_{\mathbf{3}}\right)\end{array}$ & Mean \\
\hline Tape water $\left(\mathbf{G}_{0}\right)$ & 2.1 & 2.2 & 2.2 & 2.1 \\
\hline Cow urine Conc.- $20 \%\left(\mathbf{G}_{1}\right)$ & 2.5 & 2.7 & 2.7 & 2.7 \\
\hline Cow urine Conc.- $40 \%\left(G_{2}\right)$ & 2.3 & 2.4 & 2.5 & 2.4 \\
\hline Cow urine Conc.- $60 \%\left(G_{3}\right)$ & 2.3 & 2.4 & 2.3 & 2.4 \\
\hline Cow urine Conc.- $80 \%\left(G_{4}\right)$ & 2.2 & 2.3 & 2.3 & 2.2 \\
\hline Cow urine Conc.- $100 \%\left(G_{5}\right)$ & 2.1 & 2.2 & 2.1 & 2.1 \\
\hline \multirow[t]{2}{*}{ Mean } & 2.3 & 2.3 & 2.4 & \\
\hline & Growing media $(\mathrm{P})$ & \multicolumn{2}{|c|}{ Concentration (G) } & $\mathrm{G} \times \mathrm{P}$ \\
\hline SEm \pm & 0.012 & \multicolumn{2}{|c|}{0.017} & 0.030 \\
\hline CD at $5 \%$ & 0.032 & \multicolumn{2}{|c|}{0.049} & 0.084 \\
\hline \multicolumn{5}{|c|}{ Soaking Duration } \\
\hline & $24 \mathrm{hr}\left(\mathrm{S}_{1}\right)$ & $48 \mathrm{hr}\left(\mathrm{S}_{2}\right)$ & $72 \mathrm{hr}\left(\mathrm{S}_{3}\right)$ & Mean \\
\hline Tape water $\left(\mathbf{G}_{0}\right)$ & 2.2 & 2.1 & 2.1 & 2.1 \\
\hline Cow urine Conc.- $20 \%\left(G_{1}\right)$ & 2.7 & 2.7 & 2.6 & 2.7 \\
\hline Cow urine Conc.- $40 \%\left(G_{2}\right)$ & 2.5 & 2.4 & 2.3 & 2.4 \\
\hline Cow urine Conc.- $60 \%\left(G_{3}\right)$ & 2.4 & 2.3 & 2.3 & 2.4 \\
\hline Cow urine Conc.- $80 \%\left(\mathbf{G}_{4}\right)$ & 2.3 & 2.2 & 2.2 & 2.2 \\
\hline Cow urine Conc.- $100 \%\left(\mathbf{G}_{5}\right)$ & 2.1 & 2.1 & 2.1 & 2.1 \\
\hline \multirow[t]{2}{*}{ Mean } & 2.3 & 2.3 & 2.2 & \\
\hline & Duration (S) & \multicolumn{2}{|c|}{ Concentration (G) } & $\mathrm{G} \times \mathrm{S}$ \\
\hline SEm \pm & 0.012 & \multicolumn{2}{|c|}{0.017} & 0.030 \\
\hline CD at $5 \%$ & NS & \multicolumn{2}{|c|}{0.049} & 0.084 \\
\hline
\end{tabular}


Table.4 Effect of cow urine, soaking duration and growing media on EI during 120-150 DAS

\begin{tabular}{|c|c|c|c|c|}
\hline \multirow[b]{2}{*}{ Treatment } & \multicolumn{3}{|c|}{ Growing media } & \multirow[t]{2}{*}{ Mean } \\
\hline & Without PGPR $\left(\mathbf{P}_{1}\right)$ & $\begin{array}{c}\text { With PGPR } \\
\left(\mathbf{P}_{2}\right)\end{array}$ & $\begin{array}{c}\text { With PGPR } \\
\left(\mathbf{P}_{\mathbf{3}}\right)\end{array}$ & \\
\hline Soaking duration $-24 \mathrm{hr}\left(\mathrm{S}_{1}\right)$ & 0.46 & 0.48 & 0.49 & 0.48 \\
\hline Soaking duration $-48 \mathrm{hr}\left(\mathrm{S}_{2}\right)$ & 0.43 & 0.44 & 0.45 & 0.44 \\
\hline Soaking duration $-72 \mathrm{hr}\left(\mathrm{S}_{3}\right)$ & 0.40 & 0.41 & 0.43 & 0.41 \\
\hline \multirow[t]{2}{*}{ Mean } & 0.43 & 0.44 & 0.46 & \\
\hline & Growing media (P) & \multicolumn{2}{|c|}{ Soaking duration $(\mathrm{S})$} & $\mathrm{S} \times \mathrm{P}$ \\
\hline SEm \pm & 0.001 & \multicolumn{2}{|c|}{0.001} & 0.002 \\
\hline CD at $5 \%$ & 0.003 & \multicolumn{2}{|c|}{0.003} & 0.007 \\
\hline \multicolumn{5}{|c|}{ Growing media } \\
\hline & Without PGPR $\left(\mathbf{P}_{1}\right)$ & $\begin{array}{c}\text { With PGPR } \\
\left(\mathbf{P}_{2}\right)\end{array}$ & $\begin{array}{c}\text { With PGPR } \\
\left(\mathbf{P}_{\mathbf{3}}\right)\end{array}$ & Mean \\
\hline Tape water $\left(\mathbf{G}_{\mathbf{0}}\right)$ & 0.39 & 0.40 & 0.41 & 0.40 \\
\hline Cow urine Conc.- $20 \%\left(G_{1}\right)$ & 0.48 & 0.49 & 0.50 & 0.49 \\
\hline Cow urine Conc.- $40 \%\left(G_{2}\right)$ & 0.46 & 0.47 & 0.49 & 0.47 \\
\hline Cow urine Conc.- $60 \%\left(\mathbf{G}_{3}\right)$ & 0.44 & 0.46 & 0.48 & 0.46 \\
\hline Cow urine Conc.- $80 \%\left(G_{4}\right)$ & 0.43 & 0.43 & 0.45 & 0.44 \\
\hline Cow urine Conc.- $100 \%\left(G_{5}\right)$ & 0.40 & 0.41 & 0.41 & 0.41 \\
\hline \multirow[t]{2}{*}{ Mean } & 0.43 & 0.44 & 0.46 & \\
\hline & Growing media $(\mathrm{P})$ & \multicolumn{2}{|c|}{ Concentration $(\mathrm{G})$} & $\mathrm{G} \times \mathrm{P}$ \\
\hline SEm \pm & 0.001 & \multicolumn{2}{|c|}{0.001} & 0.002 \\
\hline CD at $5 \%$ & 0.003 & \multicolumn{2}{|c|}{0.003} & 0.007 \\
\hline \multicolumn{5}{|c|}{ Soaking Duration } \\
\hline & $24 \mathrm{hr}\left(\mathrm{S}_{1}\right)$ & $48 \mathrm{hr}\left(\mathrm{S}_{2}\right)$ & $72 \mathrm{hr}\left(\mathrm{S}_{3}\right)$ & \\
\hline Tape water $\left(\mathbf{G}_{0}\right)$ & 0.42 & 0.40 & 0.38 & 0.40 \\
\hline Cow urine Conc.- $20 \%\left(\mathbf{G}_{1}\right)$ & 0.54 & 0.49 & 0.44 & 0.49 \\
\hline Cow urine Conc.- $40 \%\left(G_{2}\right)$ & 0.52 & 0.47 & 0.43 & 0.47 \\
\hline Cow urine Conc.- $60 \%\left(\mathbf{G}_{3}\right)$ & 0.50 & 0.46 & 0.41 & 0.46 \\
\hline Cow urine Conc.- $80 \%\left(\mathbf{G}_{4}\right)$ & 0.46 & 0.44 & 0.41 & 0.44 \\
\hline Cow urine Conc.- $100 \%\left(G_{5}\right)$ & 0.43 & 0.40 & 0.39 & 0.41 \\
\hline \multirow[t]{2}{*}{ Mean } & 0.48 & 0.44 & 0.41 & \\
\hline & Duration (S) & \multicolumn{2}{|c|}{ Concentration $(\mathrm{G})$} & $\mathrm{G} \times \mathrm{S}$ \\
\hline SEm \pm & 0.001 & \multicolumn{2}{|c|}{0.001} & 0.002 \\
\hline CD at $5 \%$ & 0.003 & \multicolumn{2}{|c|}{0.003} & 0.007 \\
\hline
\end{tabular}


Table.5 Effect of cow urine, soaking duration and growing media on seedling length after sowing 150 days

\begin{tabular}{|c|c|c|c|c|}
\hline \multirow[b]{2}{*}{ Treatment } & \multicolumn{3}{|c|}{ Growing media } & \multirow[t]{2}{*}{ Mean } \\
\hline & $\begin{array}{c}\text { Without PGPR } \\
\left(\mathbf{P}_{1}\right)\end{array}$ & $\begin{array}{c}\text { With PGPR } \\
\left(\mathbf{P}_{2}\right)\end{array}$ & $\begin{array}{c}\text { With PGPR } \\
\left(\mathbf{P}_{3}\right)\end{array}$ & \\
\hline Soaking duration -24 hr $\left(S_{1}\right)$ & 23.3 & 23.3 & 24.2 & 23.8 \\
\hline Soaking duration $-48 \mathrm{hr}\left(\mathrm{S}_{2}\right)$ & 21.7 & 22.1 & 22.3 & 22.0 \\
\hline Soaking duration -72 $\mathrm{hr}\left(\mathrm{S}_{3}\right)$ & 21.1 & 21.2 & 21.3 & 21.2 \\
\hline \multirow{2}{*}{ Mean } & 22.1 & 22.4 & 22.6 & \\
\hline & Growing media $(\mathrm{P})$ & \multicolumn{2}{|c|}{ Soaking duration $(\mathrm{S})$} & $\mathrm{S} \times \mathrm{P}$ \\
\hline SEm \pm & 0.355 & \multicolumn{2}{|c|}{0.355} & 0.614 \\
\hline CD at $5 \%$ & 0.994 & \multicolumn{2}{|c|}{0.994} & 1.839 \\
\hline \multicolumn{5}{|c|}{ Growing media } \\
\hline & Without PGPR $\left(\mathbf{P}_{1}\right)$ & $\begin{array}{c}\text { With PGPR } \\
\left(\mathbf{P}_{2}\right)\end{array}$ & $\begin{array}{c}\text { With } \\
\text { PGPR } \\
\left(\mathbf{P}_{\mathbf{3}}\right)\end{array}$ & Mean \\
\hline Tape water $\left(\mathbf{G}_{0}\right)$ & 20.1 & 20.2 & 20.5 & 20.3 \\
\hline Cow urine Conc.- $20 \%\left(\mathbf{G}_{1}\right)$ & 24.1 & 24.5 & 24.6 & 24.4 \\
\hline Cow urine Conc.- $40 \%\left(G_{2}\right)$ & 22.5 & 22.8 & 23.1 & 22.8 \\
\hline Cow urine Conc.- $60 \%\left(G_{3}\right)$ & 22.2 & 22.7 & 22.9 & 22.6 \\
\hline Cow urine Conc.- $80 \%\left(G_{4}\right)$ & 22.1 & 22.4 & 22.7 & 22.4 \\
\hline Cow urine Conc.- $100 \%\left(G_{5}\right)$ & 21.3 & 21.8 & 21. & 21.6 \\
\hline Mean & 22.1 & 22.4 & 22.6 & \\
\hline & Growing media $(\mathrm{P})$ & \multicolumn{2}{|c|}{ Concentration (G) } & Gx P \\
\hline SEm \pm & 0.355 & \multicolumn{2}{|c|}{0.501} & 0.868 \\
\hline CD at $5 \%$ & 0.994 & \multicolumn{2}{|c|}{1.406} & 2.260 \\
\hline \multicolumn{5}{|c|}{ Soaking Duration } \\
\hline & $24 \mathrm{hr}\left(\mathbf{S}_{1}\right)$ & $48 \mathrm{hr}\left(\mathrm{S}_{2}\right)$ & $72 \mathrm{hr}\left(\mathrm{S}_{3}\right)$ & \\
\hline Tape water $\left(\mathbf{G}_{\mathbf{0}}\right)$ & 20.9 & 20.5 & 19.4 & 20.3 \\
\hline Cow urine Conc.- $20 \%\left(G_{1}\right)$ & 29.8 & 22.1 & 21.4 & 24.4 \\
\hline Cow urine Conc.- $40 \%\left(G_{2}\right)$ & 23.5 & 22.6 & 22.3 & 22.8 \\
\hline Cow urine Conc.- $60 \%\left(G_{3}\right)$ & 23.2 & 22.5 & 22.1 & 22.6 \\
\hline Cow urine Conc.- $80 \%\left(G_{4}\right)$ & 23.1 & 22.4 & 21.6 & 22.4 \\
\hline Cow urine Conc.- $100 \%\left(\mathbf{G}_{5}\right)$ & 22.6 & 21.7 & 20.6 & 21.6 \\
\hline \multirow[t]{2}{*}{ Mean } & 23.8 & 22.0 & 21.2 & \\
\hline & Duration (S) & \multicolumn{2}{|c|}{ Concentration $(\mathrm{G})$} & Gx S \\
\hline SEm \pm & 0.355 & \multicolumn{2}{|c|}{0.501} & 0868 \\
\hline \multirow[t]{2}{*}{ CD at $5 \%$} & 0.994 & 1.40 & & 2.435 \\
\hline & \multicolumn{4}{|c|}{ Cow urine $\mathrm{x}$ soaking duration $\mathrm{x}$ growing media } \\
\hline SEm \pm & \multicolumn{4}{|c|}{1.504} \\
\hline CD at $5 \%$ & \multicolumn{4}{|c|}{4.334} \\
\hline
\end{tabular}


Table.6 Effect of cow urine, soaking duration and growing media on LAI at 120-150

\begin{tabular}{|c|c|c|c|c|}
\hline \multirow[b]{2}{*}{ Treatment } & \multicolumn{3}{|c|}{ Growing media } & \multirow[t]{2}{*}{ Mean } \\
\hline & Without PGPR $\left(\mathbf{P}_{1}\right)$ & $\begin{array}{c}\text { With PGPR } \\
\left(\mathbf{P}_{2}\right)\end{array}$ & $\begin{array}{c}\text { With PGPR } \\
\left(\mathbf{P}_{3}\right)\end{array}$ & \\
\hline Soaking duration -24 hr $\left(\mathrm{S}_{1}\right)$ & 3.7 & 3.8 & 3.8 & 3.8 \\
\hline Soaking duration $-48 \mathrm{hr}\left(\mathrm{S}_{2}\right)$ & 3.7 & 3.7 & 3.7 & 3.7 \\
\hline Soaking duration $-72 \mathrm{hr}\left(\mathrm{S}_{3}\right)$ & 3.6 & 3.7 & 3.7 & 3.7 \\
\hline Mean & 3.7 & 3.7 & 3.8 & \\
\hline & Growing media $(\mathrm{P})$ & \multicolumn{2}{|c|}{ Soaking duration $(\mathrm{S})$} & $\mathrm{S} \times \mathrm{P}$ \\
\hline SEm \pm & 0.011 & \multicolumn{2}{|c|}{0.011} & 0.018 \\
\hline CD at $5 \%$ & 0.033 & 0.0 & & 0.054 \\
\hline \multicolumn{5}{|c|}{ Growing media } \\
\hline & Without PGPR $\left(\mathbf{P}_{1}\right)$ & $\begin{array}{c}\text { With PGPR } \\
\left(\mathbf{P}_{2}\right)\end{array}$ & $\begin{array}{c}\text { With PGPR } \\
\left(\mathbf{P}_{3}\right)\end{array}$ & Mean \\
\hline Tape water $\left(\mathbf{G}_{0}\right)$ & 3.4 & 3.5 & 3.5 & 3.5 \\
\hline Cow urine Conc.- $20 \%\left(G_{1}\right)$ & 3.9 & 4.0 & 4.1 & 4.0 \\
\hline Cow urine Conc.- $40 \%\left(G_{2}\right)$ & 3.9 & 3.9 & 3.9 & 3.9 \\
\hline Cow urine Conc.- $60 \%\left(G_{3}\right)$ & 3.8 & 3.9 & 3.9 & 3.9 \\
\hline Cow urine Conc.- $80 \%\left(G_{4}\right)$ & 3.7 & 3.7 & 3.8 & 3.7 \\
\hline Cow urine Conc.- $100 \%\left(G_{5}\right)$ & 3.6 & 3.6 & 3.6 & 3.5 \\
\hline \multirow[t]{2}{*}{ Mean } & 3.7 & 3.7 & 3.8 & \\
\hline & Growing media $(\mathrm{P})$ & \multicolumn{2}{|c|}{ Concentration (G) } & Gx P \\
\hline SEm \pm & 0.011 & \multicolumn{2}{|c|}{0.015} & 0.026 \\
\hline CD at $5 \%$ & 0.033 & \multicolumn{2}{|c|}{0.042} & 0.078 \\
\hline \multicolumn{5}{|c|}{ Soaking Duration } \\
\hline & $24 \mathrm{hr}\left(\mathrm{S}_{1}\right)$ & $48 \mathrm{hr}\left(\mathrm{S}_{2}\right)$ & $72 \mathrm{hr}\left(\mathrm{S}_{3}\right)$ & \\
\hline Tape water $\left(\mathbf{G}_{0}\right)$ & 3.5 & 3.5 & 3.4 & 3.5 \\
\hline Cow urine Conc.- $20 \%\left(\mathbf{G}_{1}\right)$ & 4.0 & 3.9 & 3.9 & 4.0 \\
\hline Cow urine Conc.- $40 \%\left(G_{2}\right)$ & 3.9 & 3.9 & 3.8 & 3.9 \\
\hline Cow urine Conc.- $60 \%\left(\mathbf{G}_{\mathbf{3}}\right)$ & 3.9 & 3.9 & 3.8 & 3.9 \\
\hline Cow urine Conc.- $80 \%\left(\mathbf{G}_{4}\right)$ & 3.7 & 3.7 & 3.6 & 3.7 \\
\hline Cow urine Conc.- $100 \%\left(G_{5}\right)$ & 3.6 & 3.5 & 3.5 & 3.5 \\
\hline \multirow[t]{2}{*}{ Mean } & 3.8 & 3.7 & 3.7 & \\
\hline & Duration (S) & \multicolumn{2}{|c|}{ Concentration (G) } & $\mathrm{G} \times \mathrm{S}$ \\
\hline SEm \pm & 0.011 & \multicolumn{2}{|c|}{0.015} & 0.026 \\
\hline \multirow[t]{2}{*}{ CD at $5 \%$} & 0.042 & 0.0 & & 0.078 \\
\hline & \multicolumn{4}{|c|}{ Cow urine $\mathrm{x}$ soaking duration $\mathrm{x}$ growing media } \\
\hline SEm & \multicolumn{4}{|c|}{0.201} \\
\hline CD at $5 \%$ & \multicolumn{4}{|c|}{ NS } \\
\hline
\end{tabular}


Survival percentage of seedlings at $\mathbf{1 5 0}$ days after sowing

The results demonstrated that the effect of organic sources showed the significant effect on seedling survival percent at 150 days after sowing. The mean survival percentage of seedlings (77.0\%) was recorded under $\mathrm{G}_{1} \mathrm{~S}_{1} \mathrm{P}_{3}$ Whereas, the minimum survival percentage of seedlings $(52.50 \%)$ was recorded under control $\left(\mathrm{G}_{0} \mathrm{~S}_{3} \mathrm{P}_{1}\right)$. The result has been reported by Ambika and Balakrishanan (2015) and Parmar et al., (2016) reported that the cow urine, PGPR are well known for better germination, seedling growth, vigour and suitable for commercial use for seed soaking before sowing to improve germination and seedling health.

On the basis of present investigation, it is concluded that the $\mathrm{G}_{1}$ (20\% cow urine), $\mathrm{S}_{1} 24$ hrs seed soaking duration and $\mathrm{P}_{3}(6 \%)$ PGPR inoculation were superior over all other treatments in relation to all the viz; germination, growth and physiological parameters and the minimum results found in control in relation to all the growth parameters. Cow urine significantly encouraged germination, growth and survival of Custard apple seedlings. The interaction effect of cow urine, soaking duration and growing media showed non-significant effect on most of growth and physiological parameters of custard apple. It was concluded that $\mathrm{G}_{1} \mathrm{~S}_{1}$ combination [seed soaked under cow urine concentration $20 \%$ for $24 \mathrm{hrs]}$ was the best combination with respect to growth and survival of custard seedling.

\section{References}

Ambika S and Balakrishnan K. 2015. Enhancing germination and seedling vigour in cluster Bean by organic priming. Academic Journals 10(8): 298301.
Anonymous 2018. National Horticulture Board.

Desai, J D.1998. Seed treatment with cattle dung and GA3, and their effect on germination and subsequent growth of seedlings of Khirni. M.Sc. Thesis GAU.

Dilrukshi HNN and Perera ANF. 2009. Evaluation of an ancient technique to diagnose the pregnancy in cattle using urine. Wayamba Journal of Animal Science 6-8

Gurung N, Swamy GSK, Sarkar SK and Ubale NB. 2014. Effect of chemicals and growth regulators on germination, vigour and growth of passion fruit (Passiflora edulis Sims.). The Bioscan 9(1):155157.

Krishnamoorthy RV and Vajranabhaina SN. 1986. Biological activity of earthworm casts, An assessment of plant growth promotes or levels in the casts. Proceedings of Indian Academy of Sciences (Animal science) 95(3): 341351

Maddonni GA and Otegui ME. 1996. Leaf area, light interception and crop development in maize. Field Crops Research 48(1): 81-87.

Mane SB, Jaiswal SB, Parse and Naglot UM. 2018. Effect of pre-sowing Treatments on seed germination and growth in custard apple (Annona squamous L.) Int. J. Curr. Microbial. App. Sci. 2018. Special issues-6:1744-1748.

Parmar RK, Patel MJ, Thakkar RM and Tsomu T.2016. Influence of seed priming treatments on germination and seedling vigour of custard apple (Annonas qoumosa L.) cv. Local. An International Quarterly Journal of Life Sciences 11(1): 389-393.

Rao SP. 1975. Effect of seed treatment with cow urine on seed germination and seedling growth of custard apple. Indian Journal of Agriculture Research 9(3): 121-126. 
Ratan PB and Reddy YN. 2004. Influence of gibberellic acid on custard apple (Annonas quamosa $\quad$ L.) seed germination and subsequent seedling growth. Journal of Research ANGRAU 32(2): 93-95.

Robertson, H.M. Setten K and KoekNoorman. 1992. Fruits and seeds of
Annonaceae: Morphology and its significance for classification and identification. Bibliot. Botan. 142, 1101

Shrivastava, S.S. 2014. Horticulture Nursery: Planning and Techniques. Central Book House, Sadar Bazar Raipur, Chattisgarh.

\section{How to cite this article:}

Deeksha, T. R. Sharma, U. K. Chanderia and Dwivedi, B.S. 2020. Effect of Different Pre Sowing Treatments on Seed Germination and Growth of Custard Apple (Annona squamosa L.) Seedlings. Int.J.Curr.Microbiol.App.Sci. 9(11): 1806-1818.

doi: https://doi.org/10.20546/ijcmas.2020.911.214 